\title{
Effect of a Purified Extract of Olive Mill Waste Waters on Endothelial Cell Proliferation, Apoptosis, Migration and Capillary-Like Structure in vitro and in vivo
}

\author{
Teresa Rossi ${ }^{1}$, Barbara Bassani ${ }^{2}$, Cristina Gallo ${ }^{1}$, Sally Maramotti ${ }^{1}$, Douglas M Noonan ${ }^{2,3}$, Adriana Albini ${ }^{1 *}$ and Antonino Bruno ${ }^{2}$
}

${ }^{1}$ Laboratory of Translational Research, Department of Research and Statistics, IRCCS Arcispedale Santa Maria Nuova, Reggio Emilia, Italy

${ }^{2}$ Scientific and Technology Park, IRCCS MultiMedica, Milan, Italy

${ }^{3}$ Department of Biotechnology and Life Sciences, University of Insubria, Varese, Italy

\begin{abstract}
Olive oil, a major feature of the Mediterranean diet, is an important source of phenolic compounds. Poliphenols are associated with inhibition of several pathological processes, including cancer. Soluble phenolic compounds are contained in the aqueous part of olive and are discarded during oil production in the 'olive mill wastewaters'. Here we investigated the properties of a purified extract of olive mill wastewaters, named A009, as potential antiangiogenic compound. While the strong anti-oxidant activity of olive derived phenolic compounds has been well characterized, little is known about their anti-angiogenic properties. We investigated effects of A009 on endothelial cell morphogenesis, proliferation, migration and apoptosis, comparing the results obtained with the activity of a well-characterized olive oil phenol, Hydroxytyrosol (HT). Further, we tested the effects of A009 and HT in an in vivo angiogenesis assay. We found that A009 exerted strong anti-angiogenic effects both in vitro and in vivo, and that the complex natural purified extract has stronger anti-angiogenic potential when compared to the same concentrations of $\mathrm{HT}$ in most of the assays performed. These data demonstrate that a novel purified, polyphenol enriched extract with anti-angiogenic, and angiopreventive potential can be obtained from olive oil mill waste material, recovering useful products from an agricultural waste.
\end{abstract}

Keywords: Angiogenesis; Olive mill wastewaters; Olive oil; Hydroxytyrosol; Angioprevention; Phenols

\section{Introduction}

Olive oil represents a common component of the 'Mediterranean Diet' (MD), which has been extensively reported, along with healthy lifestyle habits, to be associated with decreased chronic conditions [1], including diabetes [2], cardiovascular diseases (CV) [3] and cancer [4]. Several epidemiological studies have shown that the incidence of several tumors is relatively low in areas where the Mediterranean diet dominates as compared to other European or North America countries [5-8].

Olive oil contains a phenolic fraction that has been reported to exert chemopreventive effects [9]. Chemoprevention [10] is the concept of preventing cancer by the administration of bioactive molecules to block, revert or delay the carcinogenic process [11]. These mechanisms include angiogenesis inhibition [12] and induction of apoptosis [13]. Among the phenols of olive oil, the most studied is hydroxytyrosol (HT) [14]. The biological properties of HT, including anti-oxidant [15], anti-apoptotic [16], anti-tumor [17] and anti-inflammatory activities [18], have been recently demonstrated by several in vitro and in vivo studies and have also been recognized by the European Food Safety Authority (EFSA).

Most of the studies concerning the benefits associated with olive oil consumption have been focused on the final product. Several processes are required for the production of high quality olive oil, which are in turn associated with waste management at each production stage. Among the waste products derived from oil production, an increased interest has been addressed to the olive-derived aqueous liquor, termed olive mill wastewater (OMWW). This waste product is highly enriched in phenol content [19], and increasing attention has been addressed to test OMWW as a source of promising bioactive compounds. The crucial points regarding the potential use of OMWW as nutraceutical, are associated with diverse factors, including the type and maturation state of olives, agricultural region, climatic conditions, related agrotechnology/processing methods and oil extraction procedures [20]. These features are crucial in determining the OMWW phenolic fraction composition, which has been observed to range from 0.5 to $24 \mathrm{~g} / \mathrm{L}$ [21]

While the anti-tumor effects of the phenolic compounds are largely studied [22], little is known about their anti-angiogenic and angiopreventive potential referred as the ability to target angiogenesis in cancer prevention [23]. Angiogenesis, the formation of new blood vessels from preexisting vasculature, is essential for many physiological processes, such as embryogenesis, and also plays a critical role in many pathological conditions, including cancer. This process is an essential event for the tumor growth and a crucial hallmark of cancer progression and metastasis [24]. Several diet derived compounds and their synthetic derivatives have been reported to exert anti-oxidant, anti-tumor [25], anti-angiogenic and angiopreventive activities [26] in a wide array of hematologic and solid malignancies.

Therefore, the aim of the present study was to evaluate the antiangiogenic and angiopreventive potential of an highly phenol-rich olive mill wastewater purified extract, termed A009, on endothelial cell

*Corresponding author: Adriana Albini, IRCCS, Advanced Technologies and Healthcare Models in Oncology, Arcispedale S Maria Nuova, Department of Research and Statistics, Viale Umberto I, 50 - 42123 Reggio Emilia, Tel: +0522 295645; E-mail: albini.adriana@gmail.com

Received April 01, 2015; Accepted June 02, 2015; Published June 04, 2015

Citation: Rossi T, Bassani B, Gallo C, Maramotti S, Noonan DM, et al. (2015) Effect of a Purified Extract of Olive Mill Waste water on Endothelial Cell Proliferation, Apoptosis, Migration and Capillary-Like Structure in vitro and in vivo. J Bioanal Biomed S12: 006. doi:10.4172/1948-593X.S12-006

Copyright: $\odot 2015$ Rossi T, et al. This is an open-access article distributed under the terms of the Creative Commons Attribution License, which permits unrestricted use, distribution, and reproduction in any medium, provided the original author and source are credited. 
Citation: Rossi T, Bassani B, Gallo C, Maramotti S, Noonan DM, et al. (2015) Effect of a Purified Extract of Olive Mill Waste water on Endothelial Cell Proliferation, Apoptosis, Migration and Capillary-Like Structure in vitro and in vivo. J Bioanal Biomed S12: 006. doi:10.4172/1948-593X. S12-006

Page 2 of 8

proliferation, induction of apoptosis, migration and network formation activities in vitro and its ability to interfere with angiogenesis in vivo. Furthermore, we demonstrate that the A009 phenol-rich extract exerts a more potent angiopreventive/anti-angiogenic activity as compared to purified HT alone. Our results underline the possibility to derive promising bioactive compounds from an agricultural waste product, providing a valid dietary supplement while containing waste product.

\section{Materials and Methods}

\section{Reagents}

OMWW were kindly provided by Agriturismo La Vialla (Castiglion Fibocchi (Arezzo), Italy). A009 a phenol rich purified extract was obtained from Massimo and Daniele Pizzichini according to Patent formulation (Patent 8815815). All experiments were performed using 2 different A009 batches. This is an important control to insure activity is not altered in different batches due to potential variations in chemical composition and compounds present in the extract that might be influenced by certain conditions including seasonal weather variations and the times between olive oil extraction and extract preparation. Hydroxytyrosol (HT), synthetic in origin whose purity is $\geq 98 \%$, was purchased from Cayman Chemicals (Ann Arbor, Michigan, USA). Ethanol (EtOH, the vehicle for HT) was purchased from Sigma-Aldrich (Milan, Italy).

\section{Preparation of A009 and phenolic quantification}

Olive extract was obtained from OMWW using two sequential cross-flow filtration process. First, a ceramic Microfiltration (MF) was performed using 2 tubular membranes in alumina oxide with a $300 \mathrm{KDa}$ cut-off 3 (TAMI membranes, Nyons, France) and a filtering surface of $0.35 \mathrm{~m}^{2}$. This removes solid particles, residual plant particles and cells that were discarded. The MF permeate was further concentrated by reverse osmosis (RO) in a Polyamide spiral wound module (Microdyn Nadir, Wiesbaden, Germany) with a filtering surface of $7 \mathrm{~m}^{2}$. The RO permeate was essentially ultrapure water and was discarded. Finally, the RO concentrate, obtained reaching a volume concentration ratio (VCR) of 3.6, constituted the olive extract (here termed A009). Quantification of the phenolic presence in A009 was performed using a high-performance liquid chromatography (HPLC) analysis. Quantification revealed that the major phenolic component of A009 was hydroxytyrosol in both the batches ( 2.7 and $5.72 \mathrm{gr} / \mathrm{L}$ ), along with several other phenolic compounds (Table S1).

\section{Cell cultures}

Human umbilical vein endothelial cells (HUVECs) were purchased from Promo Cell (Heidelberg, Germany) and grown on 1\% gelatincoated tissue culture plates in Medium 199 (Sigma Aldrich Milan, Italy), supplemented with $10 \%$ heat-inactivated fetal bovine serum (FBS) (Euroclone, Milan, Italy), 1\% glutamine (Euroclone, Milan, Italy), fibroblast growth factors $(1 \mu \mathrm{g} / 100 \mathrm{ml}$ acid-fibroblast growth factor plus $1 \mu \mathrm{g} / 100 \mathrm{ml}$ basic-fibroblast growth factor, PeproTech London UK), epidermal growth factor $(1 \mu \mathrm{g} / 100 \mathrm{ml}$ PeproTech London UK), heparin (10 mg/100 ml, Sigma Aldrich Milan, Italy) and hydrocortisone $\left(0.1 \mathrm{mg} / 100 \mathrm{ml}\right.$ Sigma Aldrich Milan, Italy) at $37^{\circ} \mathrm{C}$ in $5 \% \mathrm{CO}_{2}$. Cells were used between the second and sixth passage in vitro.

\section{In vitro cell proliferation}

HUVEC proliferation was assessed by the MTT (3-(4,5-dimethylthiazol-2-yl)-2,5-diphenyltetrazolium bromide;

\begin{tabular}{|l|l|l|}
\hline Phenolic compound (g/L) & A009 batch 1 & A009 batch 2 \\
\hline Hydroxytyrosol glucoside & ND & 1,69 \\
\hline Hydroxytyrosol & 2.7 & 5.72 \\
\hline Tyrosol & 0.2 & ND \\
\hline Chlorogenic acid & 0.12 & 0.1 \\
\hline B-hydroxyverbascoside isomer 1 & 0.35 & 0.14 \\
\hline B-hydroxyverbascoside isomer 2 & 0.32 & 0.17 \\
\hline Verbascoside & 0.84 & 1.32 \\
\hline caffeyol ester of secologanoside & ND & 0,2 \\
\hline Decarboxymethyloleuropeinaglycon & 1.99 & 0.28 \\
\hline Oleouropein aglycon & ND & 0.22 \\
\hline 6'-p-coumaroyl secologanoside & ND & 0.4 \\
\hline Rutin & 0.11 & ND \\
\hline luteolin-7-o-glucoside & 0.22 & ND \\
\hline
\end{tabular}

Table S1: Phenolic quantification in OMWW extract. Quantification of the phenolic presence in $\mathrm{A} 009$ was performed using an highperformance liquid chromatography (HPLC) analysis. Results are expressed as g/L. N.D. Not detected.

Sigma Aldrich, Milan, Italy) assay. Cells ( $10^{3} /$ well $)$ were seeded into 96-multiwell plates in complete medium. After cell adhesion, fresh complete medium with decreasing dilutions of treatments was added as follows: A009 (1:10000-1:100), HT (1:10000-1:100) and EtOH (1:10000-1:100). Cells were treated for $24,48,72$ and 96 hours and the relative absorbance was measured at $540 \mathrm{~nm}$ by a FLUOstar spectrophotometer (FLUOstar Omega BMG LABTECH, Ortenberg, Germany).

\section{Apoptosis assay}

HUVECs $\left(1.5 \times 10^{5}\right.$ cells/well $)$ were plated in 6-well plates and incubated with decreasing dilutions (1:2500-1:250) of A009, HT or EtOH for $24 \mathrm{~h}$ and $48 \mathrm{~h}$ in $10 \%$ FBS-supplemented complete medium. Cells were recovered, washed twice with PBS and transferred to test tubes. Cells were pelleted by centrifugation, resuspended in Annexin V-binding buffer (BD Biosciences, Milan, Italy) and stained with Fluorescein isothiocyanate (FITC)-conjugated Annexin $\mathrm{V}$ and 7-amino-actinomycin D (7-AAD) (BD Biosciences Milan, Italy) for 15 min at $4^{\circ} \mathrm{C}$ in the dark. Cells were then washed in PBS, supernatants discarded and resuspended in $400 \mu \mathrm{L}$ of PBS. Analysis was performed by flow cytometry using a FACSCanto (BD Biosciences, Milan, Italy) on FSC/SSC viable gated cells, excluding cell debris, with excitation set at $488 \mathrm{~nm}$ and emission at $518 \mathrm{~nm}$ (FITC detector) and $620 \mathrm{~nm}$ (PE detector). Data were analyzed using FACSDiva Software 6.1.2. The experiment was performed three times and each condition was in duplicate.

\section{Induction of reactive oxygen species (ROS)}

The ability of A009 to inhibit intracellular ROS generation on treated or pre-treated endothelial cells was determined using 2,7-dichlorofluorescin diacetate (DCFH-DA; Sigma Aldrich, Milan, Italy) assay coupled with flow cytometry based on the previously described protocol [27]. The assay was performed by 2',7'-dichlorofluorescin diacetate staining ( $\left.\mathrm{H}_{2} \mathrm{DCF}-\mathrm{DA}\right)$ in order to detect and localize intracellular sources of ROS. Non-ionized $\mathrm{H}_{2} \mathrm{DCF}-$ $\mathrm{DA}$ is membrane permeant and therefore is able to diffuse readily into cells, once within the cell, the acetate groups are hydrolysed by intracellular esterase activity forming 2',7'- dichlorodihydrofluorescein $\left(\mathrm{H}_{2} \mathrm{DCF}\right)$ which is polar and thus trapped within the cell. $\mathrm{H}_{2} \mathrm{DCF}$ fluoresces when it is oxidized by $\mathrm{H}_{2} \mathrm{O}_{2}$ or lipid peroxides to yield 2',7'-dichlorofluorescein (DCF). The level of DCF produced within 
Citation: Rossi T, Bassani B, Gallo C, Maramotti S, Noonan DM, et al. (2015) Effect of a Purified Extract of Olive Mill Waste water on Endothelial Cell Proliferation, Apoptosis, Migration and Capillary-Like Structure in vitro and in vivo. J Bioanal Biomed S12: 006. doi:10.4172/1948-593X. S12-006

Page 3 of 8

the cells is related linearly to that of peroxides present and thus its fluorescent emission provides a measure of the peroxide levels.

A009 pre-treatment of endothelial cells was performed by transferring $1.5 \times 10^{5}$ HUVECs to test tubes and incubating them with decreasing dilutions (1:500-1:250) of A009, HT or EtOH in complete medium for 30 minutes at $37^{\circ} \mathrm{C}, 5 \% \mathrm{CO}_{2}$. Cells were then resuspended in PBS and incubated with $\mathrm{H}_{2}$ DCF-DA $(10 \mu \mathrm{M})$ and $\mathrm{H}_{2} \mathrm{O}_{2}(250 \mu \mathrm{M})$ for 45 minutes at $37^{\circ} \mathrm{C}, 5 \% \mathrm{CO}_{2}$. The fluorescence intensity $(525 \mathrm{~nm})$ was detected using a FACSCanto (BD Biosciences, Milan, Italy) flow cytometer.

For $\mathrm{H}_{2} \mathrm{O}_{2}$ pre-treatment, $1.5 \times 10^{5}$ HUVECs were transferred to test tubes in complete medium and incubated with $\mathrm{H}_{2} \mathrm{O}_{2}(250 \mu \mathrm{M})$ for 15 minutes at $37^{\circ} \mathrm{C}$ and $5 \% \mathrm{CO}_{2}$. Cells were then resuspended in PBS and incubated with $\mathrm{H}_{2}$ DCF-DA $(10 \mu \mathrm{M})$ and decreasing dilutions (1:500-1:250) of A009, HT or EtOH for 45 minutes at $37^{\circ} \mathrm{C}$ and $5 \%$ $\mathrm{CO}_{2}$. The fluorescence $(525 \mathrm{~nm})$ was measured using a FACSCanto (BD Biosciences, Milan, Italy) flow cytometer.

\section{Migration and invasion assays}

Chemoinvasion and chemotaxis assays were performed using modified Boyden chambers, as previously described [28,29]. HUVECs $\left(5 \times 10^{4}\right)$ were pre-treated with decreasing dilutions (1:500-1:250) of $\mathrm{A} 009, \mathrm{HT}$ or EtOH as a vehicle for $24 \mathrm{~h}$, then washed in PBS, resuspended in serum-free medium and placed in the upper compartment of the chamber. M199 medium supplemented with 10\% FBS as attractant factor was added in the lower chamber compartment. $8 \mu \mathrm{m}$ poresize polycarbonate filters (Whatman, GE Healthcare Europe GmbH, Milan, Italy) were pre-coated with collagen IV $(50 \mu \mathrm{g} / \mathrm{mL}$, Sigma Aldrich, Milan, Italy) for the chemotaxis assay or matrigel $(1 \mathrm{mg} / \mathrm{mL}$, BD Biosciences, Milan, Italy) for the chemoinvasion assay. Cells were incubated for 6 (chemotaxis) or 24 (chemoinvasion) hours at $37^{\circ} \mathrm{C}$, filters were then recovered, cells on the upper surface mechanically removed with a cotton swab and cells migrated or invaded toward the lower filter surface fixed with absolute ethanol and stained with DAPI (Vectashield, Vector Laboratories, Orton Southgate, Peterborough, United Kingdom). Cells were counted in a double-blind manner in five consecutive fields with a fluorescent microscope (Nikon Eclipse Ni). All experiments were performed three times in duplicate.

\section{Morphogenesis assay on matrigel}

The ability of A009, HT or EtOH to interfere with the capillarylike network formation was assessed by the morphogenesis assay in vitro [30]. A 24 -well plate, pre-chilled at $-20^{\circ} \mathrm{C}$, was carefully filled with $300 \mu \mathrm{L}$ per well of liquid matrigel (BD Biosciences, Milan, Italy) at $4^{\circ} \mathrm{C}$ with a pre-chilled pipette, avoiding bubbles and polymerized for $1 \mathrm{~h}$ at $37^{\circ} \mathrm{C}$. HUVECs $\left(5 \times 10^{4}\right.$ cells/well $)$ were suspended in $1 \mathrm{~mL}$ of complete medium supplemented with $10 \%$ FBS in the presence of decreasing dilution (1:500-1:250) of A009, HT or EtOH and carefully layered on the top of the polymerized matrigel. Effects on the growth and morphogenesis of HUVECs were recorded after $6 \mathrm{~h}$ incubation with an inverted microscope (Nikon Eclipse TS100). The number of segments and their length were quantified using ImageJ software and the "Angiogenesis Analyzer" tool.

\section{In vivo matrigel sponge assay}

The ability of A009 to inhibit angiogenesis in vivo was assessed by the matrigel sponge assay, as already described [31]. A009 or HT (dilution 1:500 and 1:250) were added to unpolymerized liquid matrigel, containing an heparin $(25 \mathrm{U} / \mathrm{mL})$, VEGF $(100 \mathrm{ng} / \mu \mathrm{L})$ and
TNF- $\alpha(1.2 \mathrm{ng} / \mu \mathrm{L})$ mixture (VTH), and slowly subcutaneously injected into the flanks of 6- to 8-week-old C57/BL6 male mice (Charles River Laboratories, Calco (Lecco), Italy) in groups of 4 animals for each treatment. The animal care and experimental protocols were performed according to the Italian Approved Animal Welfare Assurance. Following four days after injection, mice were sacrificed and the pellets were removed, weighted, and cryostored using OCT for histological examination, or minced and diluted in PBS for hemoglobin content measurement. Hemoglobin content was determined using $200 \mu \mathrm{L}$ of the supernatant derived from minced pellets, centrifuged for 12 minutes $13000 \mathrm{~g}$ at $4^{\circ} \mathrm{C}$ and mixed with $800 \mu \mathrm{L}$ of Drabkin reagent (Sigma Aldrich, Milan, Italy). The final hemoglobin concentration was calculated from a standard calibration curve, by spectrophotometric analysis at $540 \mathrm{~nm}$ (FLUOstar Omega BMG LABTECH, Ortenberg, Germany). The hemoglobin concentration was calculated as: (Abs 540 $\mathrm{nm} /$ pellet weight $\mathrm{mg}) \times 100$.

\section{Immunohystochemical analysis}

Sections of $5 \mu \mathrm{m}$ thickness from OCT cryostored samples were stained with hematoxylin and eosin for histological examination following standard procedures. The sections were photographed on an inverted microscope (Nikon Eclipse TS100).

\section{Statistical analysis}

Results are showed as mean \pm SD. The significance of differences was evaluated with a two-tailed t-test using Prism software (GraphPad Software for Science, Inc., San Diego, CA, USA).

\section{Results}

\section{A009 inhibits HUVECs proliferation}

A009 was tested for its ability to interfere with endothelial cell proliferation, and compared with HT alone at a similar concentration $(2.7 \mathrm{~g} / \mathrm{L})$ as that contained for A009 (Table S1). A009 was able to inhibit HUVEC proliferation starting from 24 hours at 1:1000 dilution, while higher concentrations of A009 completely arrested cell proliferation (Figure 1A). When compared to a similar dilution of HT alone, HT showed a lesser effect on cell proliferation, suggesting that the presence of heterogeneous complex of phenolics in the A009 extract enhances anti-proliferative activity. Finally, EtOH, the vehicle for HT, showed no effect on HUVEC proliferation (data not shown). The IC50 of A009 was determined using the concentration of HT presented in the pool as shown in Figure 1B.

\section{A009 induces apoptosis on endothelial cells}

We evaluated whether the cytostatic effect of A009 was associated with the induction of apoptosis using a flow cytometry based apoptosis assay. Following 24 hours of treatment with A009 (1:500 and 1:250) $30 \%$ and $50 \%$, respectively, of HUVECs had entered into apoptosis, as detected by AnnexinV and 7-AAD positivity (Figure $2 \mathrm{~A}$ ). In contrast, HUVECs exposed to HT alone, at the same dilution ranges (1:2500$1: 250)$ as those for A009, showed only low apoptotic rates (15\% of Annexin $\mathrm{V}^{+} 7-\mathrm{AAD}^{+}$cells at 1:250 dilution). After 48 hours of treatment, HUVECs showed increased apoptosis with A009 as compared to HT alone (Figure 2B). Cells treated with vehicle showed very low apoptosis.

\section{A009 inhibits ROS production before and after $\mathrm{H}_{2} \mathrm{O}_{2}$ treatment}

Oxidative stress occurs when disequilibrium between reactive 
Citation: Rossi T, Bassani B, Gallo C, Maramotti S, Noonan DM, et al. (2015) Effect of a Purified Extract of Olive Mill Waste water on Endothelial Cell Proliferation, Apoptosis, Migration and Capillary-Like Structure in vitro and in vivo. J Bioanal Biomed S12: 006. doi:10.4172/1948-593X. S12-006

Page 4 of 8

oxygen species (ROS) production and host antioxidant capacity is present. Reactive Oxygen Species are induced as byproducts during the mitochondrial electron transport of aerobic respiration or by oxide-reductase enzymes and metal catalyzed oxidations, and are associated with several inflammatory conditions. Given the antioxidant compounds present in A009, we evaluated the scavenger ability of A009 on HUVECs following pre-treatment with $\mathrm{H}_{2} \mathrm{O}_{2}$ or A009, as compared to HT alone. A009 showed strong ROS scavenger effects, significantly decreasing the $\mathrm{H}_{2} \mathrm{DCF}-\mathrm{DA}$ fluorescence signal, both in pre-treatment (Figure $3 \mathrm{~A}, \mathrm{p}<0.001$ for $1: 500$ and 1:250 dilution), and post-treatment (Figure 3B $\mathrm{p}<0.001$; for $1: 500$ and 1:250 dilutions). HT alone exerted little ROS scavenger activities, supporting the hypothesis of an enhanced anti-oxidant effect in A009.

\section{A009 inhibits endothelial morphogenesis in vitro}

When cultured on matrix layer supplemented with pro-angiogenic factors, HUVECs are able to organize into capillary-like networks. We observed that A009 was able to interfere with HUVECs morphogenesis in a dose-dependent manner to a similar extent as HT alone (Figure $4 \mathrm{~A})$. The quantification of the number of meshes (Figure $4 \mathrm{~B}$ ) indicated a statistically significant effect of both A009 $(1: 250 \mathrm{p}<0.05)$ and HT $(1: 250 \mathrm{p}<0.05)$.
A)

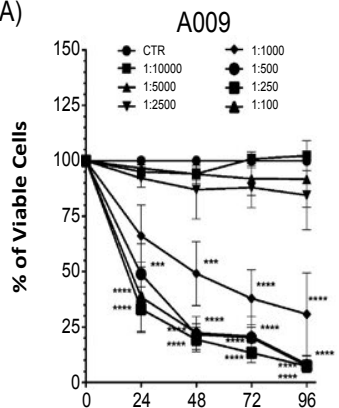

TIME (HOURS)

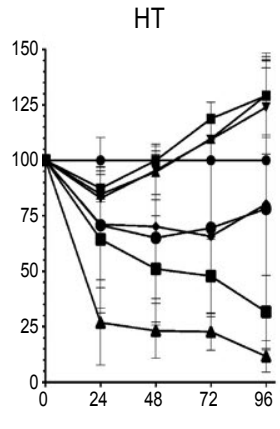

TIME (HOURS)
B)

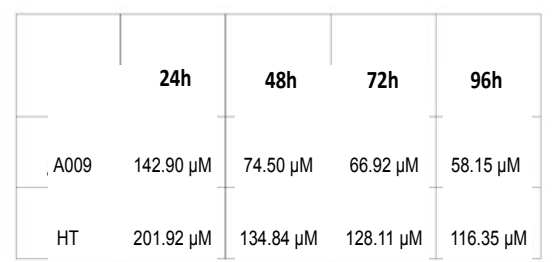

Figure 1: Anti-proliferative properties of A009.

HUVE cells were plated at low density (1000 cells/well) into 96-multiwell plates in M199 complete medium and treated with increasing dilutions of A009, hydroxytyrosol alone (HT) or ethanol (data not shown). Cell proliferation was evaluated at determined times $(24,48,72,96 \mathrm{~h})$ using the MTT (3-(4,5-dimethylthiazol-2-yl)-2,5-diphenyltetrazolium bromide) assay. 009 inhibited cell proliferation in a dose-dependent manner; the inhibitory effect of A009 was already significant after $24 \mathrm{~h}$ of treatment with a dose of 1:500, whereas $\mathrm{HT}$ alone showed no significant inhibitory effect $(A)$. We calculated the IC50 treatment in order to define the effectiveness of A009 and HT in inhibiting HUVE cells proliferation. A009 showed lower IC50 value as compared to HT alone, demonstrating the grater effectiveness of the purified extract $(B)$.

Mean \pm SD of 3 independent experiments is shown. Student's t-test was used to determine pvalue $\left({ }^{* * *} p<0.001 ;{ }^{* * * *} p<0.0001\right)$

\section{A009 inhibits HUVECs migration and invasion}

Endothelial cells must cross basement membranes to form new blood vessels during angiogenesis. We therefore investigated whether the A009, HT or EtOH were able to affect HUVEC migration and invasion through matrigel, a reconstituted basement membrane [28,29]. A009, at 1:500 and 1:250 dilutions, significantly decreased the number of migrated (Figure $5 \mathrm{~A}, \mathrm{p}<0.01$ ) and invaded (Figure $5 \mathrm{~B}$, $\mathrm{p}<0.0001)$ endothelial cells in a dose-dependent manner. A significant migration and invasion inhibitory effect with HT was observed only at the highest concentration.

\section{A009 inhibits in vivo angiogenesis}

We assessed the effect of A009 and $\mathrm{HT}$ on in vivo angiogenesis using a rapid and quantitative subcutaneous matrigel sponge assay [31]. A cocktail of VEGF, TNF- $\alpha$ and heparin promoted an hemorrhagic

HUVEC $24 h$

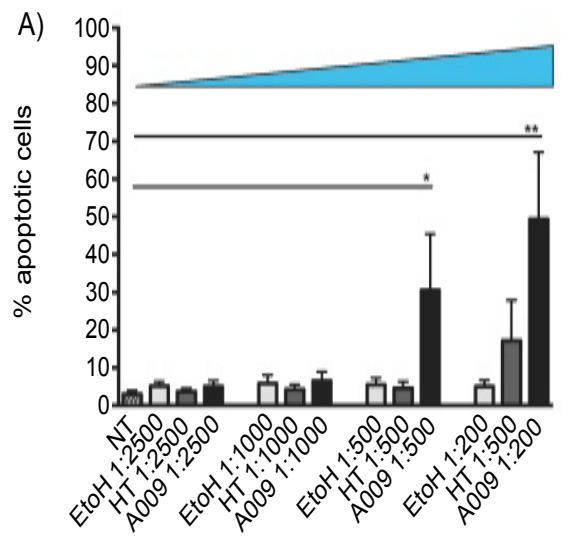

HUVEC 48h

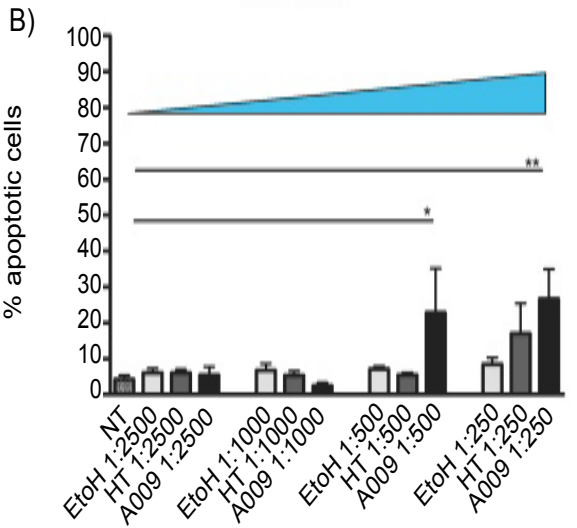

Figure 2: Induction of apoptosis by A009.

In the apoptosis assay, HUVECs $\left(1.5 \times 10^{5}\right.$ cells/well) were plated onto six-well plates and were treated with decreasing dilutions (1:2500-1:250) of A009 or HT for $24 \mathrm{~h}$ (A) and $48 \mathrm{~h}$ (B) in complete M199 medium. Vehicle (ethanol) and M199 medium alone were used as negative and positive controls, respectively. Fluorescence signal for Annexin V and 7 AAD (7amino-actinomycin D) was used to detect viable, dead or apoptotic cells; the fluorescence signal was measured with a FACSCanto flow cytometer and data were analyzed with the FACSDiva Software 6.1.2. After $24 \mathrm{~h}$ of treatment with A009, HUVECs underwent apoptosis in a dose-dependent manner, while $\mathrm{HT}$ induced apoptosis only at the highest concentration (1:250). Mean \pm SD of 3 independent experiments is shown. Student's t-test was used to determine p-value. $\left({ }^{*} p<0.05^{* *} p<0.01\right)$ 
Citation: Rossi T, Bassani B, Gallo C, Maramotti S, Noonan DM, et al. (2015) Effect of a Purified Extract of Olive Mill Waste water on Endothelial Cell Proliferation, Apoptosis, Migration and Capillary-Like Structure in vitro and in vivo. J Bioanal Biomed S12: 006. doi:10.4172/1948-593X. S12-006

A)

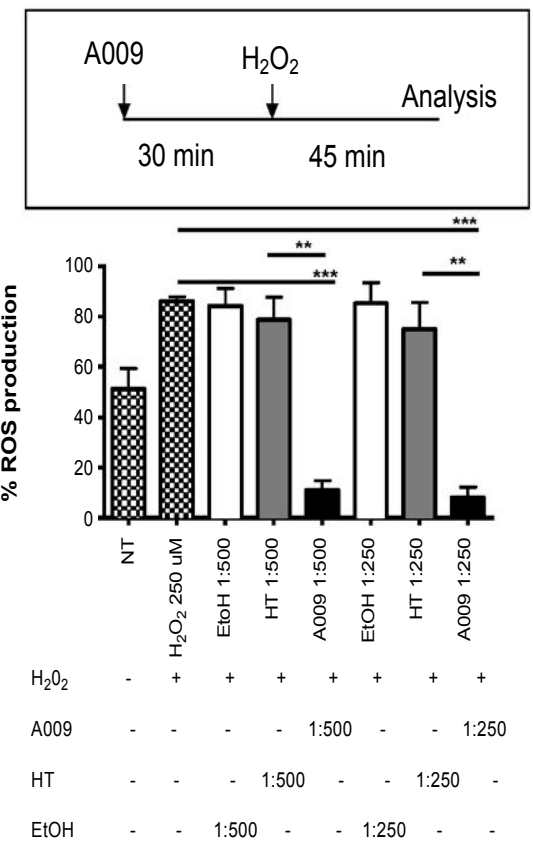

B)

\begin{tabular}{|lll}
$\mathrm{H}_{2} \mathrm{O}_{2}$ & $\mathrm{~A} 009$ \\
$15 \mathrm{~min}$ & \multicolumn{2}{|c|}{ Analysis } \\
\hline
\end{tabular}

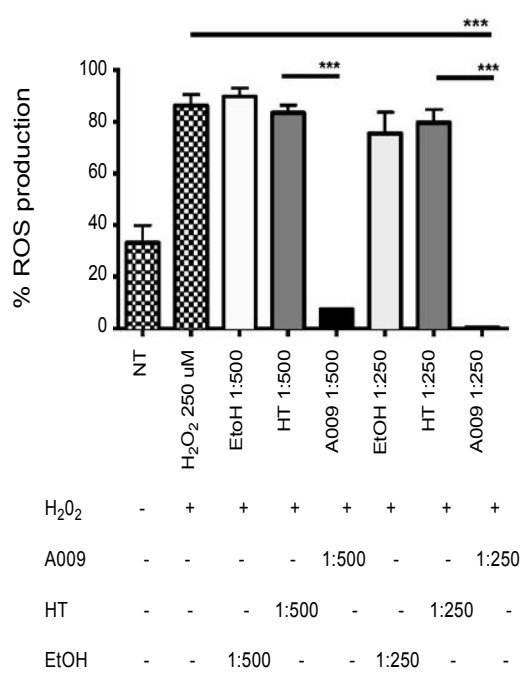

Figure 3: Anti-oxidative effects of A009

The ability of A009 to interfere with the induction of reactive oxygen species (ROS) was assessed by staining with DCFH-DA followed by flow cytometry analysis. HUVECs $\left(1.5 \times 10^{5}\right.$ cells/treatment $)$ were pre-treated with decreasing dilution (1:500 and 1:250) of $\mathrm{A} 009, \mathrm{HT}$ and $\mathrm{EtOH}$, then followed by exposure to $250 \mu \mathrm{M} \mathrm{H}_{2} \mathrm{O}_{2}$ (A) or pretreated with $250 \mu \mathrm{M} \mathrm{H}_{2} \mathrm{O}_{2}$ followed by treatment with decreasing dilution (1:500 and 1:250) of $\mathrm{A009}, \mathrm{HT}$ and $\mathrm{EtOH}(\mathrm{B})$, to assess the scavenger activity in a prevention and an intervention schedule, respectively. Fluorescence was measured with FACSCanto and data analyzed with the FACSDiva Software 6.1.2. A009 was able to inhibit the ROS production in both schedules in a dose-dependent manner with more efficacy than HT alone. Complete medium (NT, medium M199 supplemented with 10\% FBS) and $\mathrm{H} 2 \mathrm{O} 2(250 \mu \mathrm{M})$ were used as negative and positive control, respectively. Mean \pm SD of 3 independent experiments is shown. Student's t-test was used to determine $p$-value. $\left({ }^{* *} p<0.01 ;{ }^{* * *} p<0.001\right)$.
A)
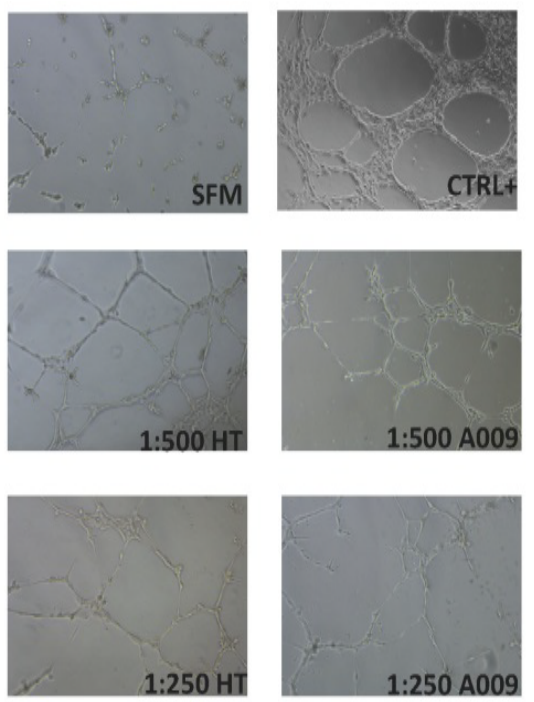

B)

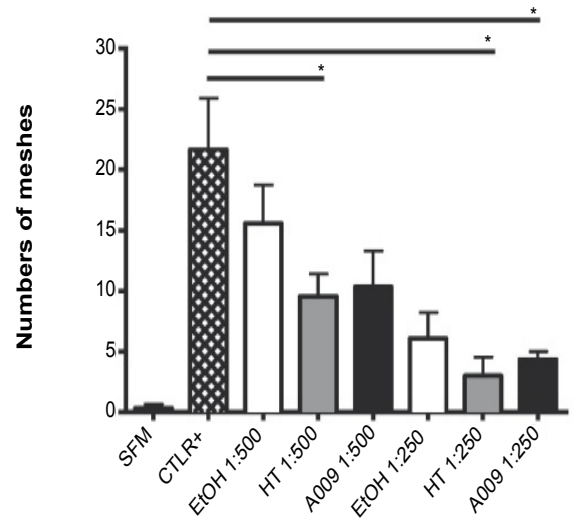

Figure 4: Effects of A009 on endothelial cell morphogenesis.

The anti-angiogenic activity of A009 and HT alone were tested in HUVECs using a morphogenesis assay. HUVECs $\left(5 \times 10^{4}\right.$ cells/well) were plated on a 24-multiwell plate,precoated with $10 \mathrm{mg} / \mathrm{ml}$ extracellular membrane basement matrix (Matrigel), then treated for 6 hours with decreasing dilutions (1:5001:250) of A009, HT (A) or EtOH (data not shown) in complete M199 medium. Complete medium (CTRL+, M199 supplemented with 10\% FBS) and serumfree medium (SFM) were used as positive and negative controls, respectively. The number of meshes were quantified with the ImageJ software "Angiogenesis Analyzer" tool (B). A009 and HT were both able to inhibit morphogenesis in an equivalent manner. Mean \pm SD of 3 independent experiments is shown. Student's t-test was used to determine $p$-value $\left({ }^{*} p<0.05\right)$

vascularization of the matrigel sponge, which was detectable at 4 days post-implantation. Addition of A009 (1:500 and 1:250) significantly inhibited the VTH-induced angiogenic response (1:500 $\mathrm{p}=0.0058$ and 1:250 $\mathrm{p}=0.0189)$ as detected by macroscopic inspection of the pellets (Figure 6A). Quantification using the colorimetric Drabkin's assay for hemoglobin indicated a statistically significant inhibition of angiogenesis by A009 (Figure 6B). Interestingly, HT showed limited effects on angiogenesis in vivo. Hematoxylin and eosin stained sections of explanted-matrigel pellets confirmed the inhibition of angiogenesis process after A009 treatment (Figure 6C).

\section{Discussion}

There is a growing interest in finding novel active compounds 
Citation: Rossi T, Bassani B, Gallo C, Maramotti S, Noonan DM, et al. (2015) Effect of a Purified Extract of Olive Mill Waste water on Endothelial Cell Proliferation, Apoptosis, Migration and Capillary-Like Structure in vitro and in vivo. J Bioanal Biomed S12: 006. doi:10.4172/1948-593X. S12-006

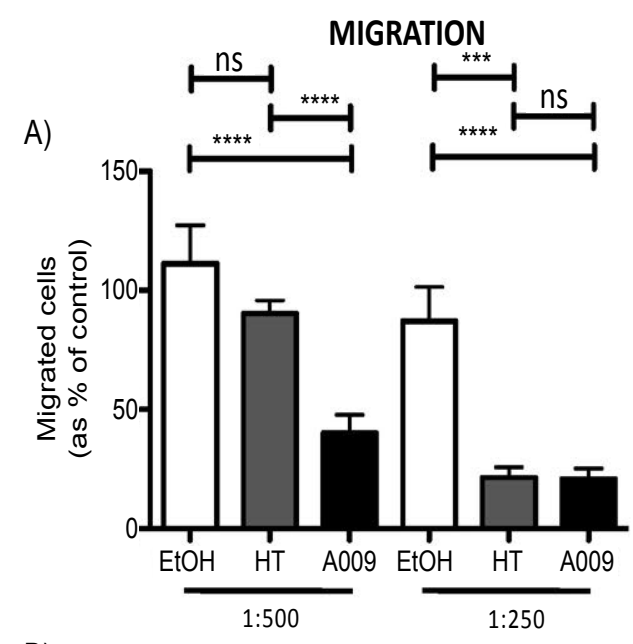

B)

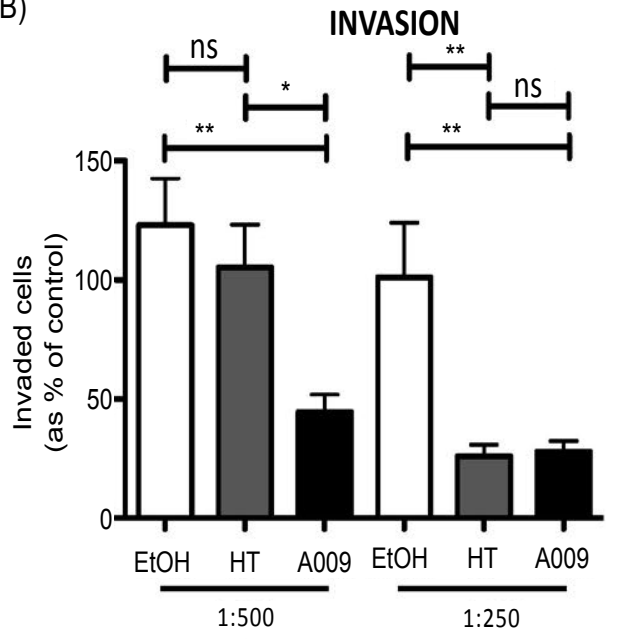

Figure 5: Effects of A009 on endothelial cell migration and invasion. The ability of A009 to interfere with HUVEC migration (A) and invasion (B) were assessed using a modified Boyden chamber assays. HUVECs were pretreated for $24 \mathrm{~h}$ with decreasing dilutions of A009, HT or EtOH (1:500-1:250), then the HUVECs were seeded in the upper compartment of Boyden chamber in serum-free medium. Complete medium (M199 supplemented with 10\% FBS) or serum-free medium was placed in the lower compartment.

Following 6 or 24 hours of incubation for the assessment of migration or invasion respectively, the migrated/invaded cells were fixed, stained and counted. A009 was able to inhibit migration and invasion at lower concentrations as compared to HT alone. Mean \pm SD of 3 independent experiments is shown. Student's t-test was used to determine $p$-value $\left({ }^{* * *} p<0.001 ;{ }^{* * *} p<0.0001\right)$ for migration and $\left({ }^{*} p<0.05 ;{ }^{* *} p<0.01\right)$ for invasion.

derived from food and beverage sources [32] in relation to their biological properties and potential health benefits. Increased attention has been addressed to polyphenolic compounds, as a consequence of their anti-proliferative, anti-oxidant and anti-angiogenic properties $[12,33]$. Olive oil is a basic feature of Mediterranean diet that has been associated with diverse health effects and several studies have investigated the bioactive properties of its components [22,34]. Soluble phenolics compounds present in the olive fruit are discarded during production of olive oil in the form of OMWW. Given the concerns associated to waste product management, some studies addressed their attention to OMWW, identifying several compounds contained in this waste product that have been reported to be rich in phenolics [19]. Regarding the chemical composition of OMWW, HT has been
A)

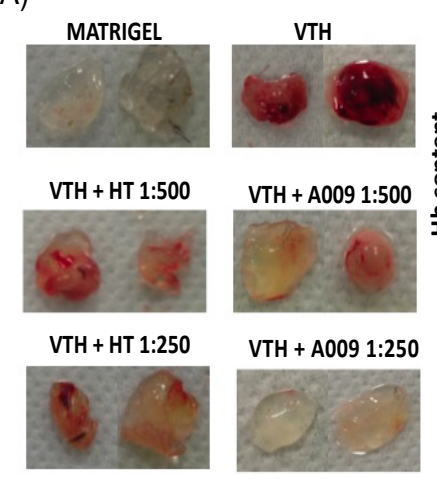

B)

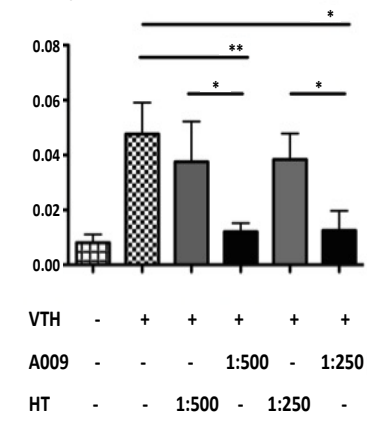

C)
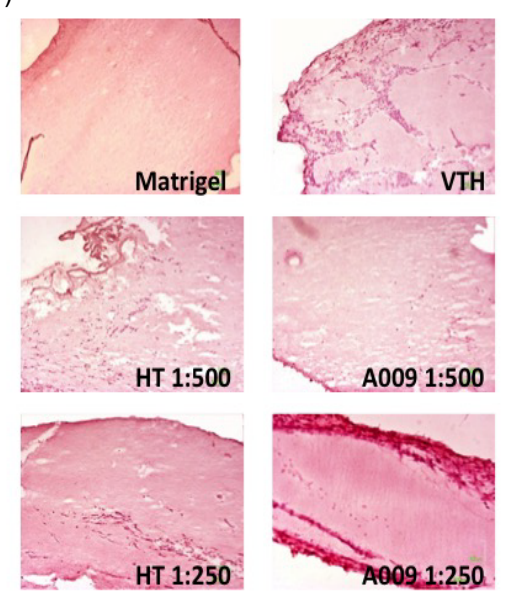

Figure 6: Anti-angiogenic activities of A009 in vivo.

Matrigel containing a pro angiogenic mixture (VTH: VEGF, TNF- $\alpha$, heparin), with or without either $\mathrm{A} 009$ or $\mathrm{HT}$ alone, were subcutaneously injected into the flanks of mice. The Matrigel pellets were excised 4 days after injection, recovered, observed for macroscopic red coloration (A) and assessed for hemoglobin $(\mathrm{Hb})$ content using the colorimetric Drabkin's assay $(B)$. Hematoxylin and eosin staining of explanted matrigel pellets $(\mathrm{C})$ were performed to evaluate the presence of total cell infiltrate. A009 was more effective than HT alone in inhibiting the angiogenic process. Mean \pm SD is shown, $\left({ }^{*} p<0.05 ;{ }^{* \star} p<0.01\right)$.

reported as a major component [14], which has been demonstrated to exert anti-oxidant, anti-inflammatory and anti-tumor effects [15-17]. We investigated the properties of a novel purified polyphenol-enriched extract of OMWW abundant in HT consistently with the previous literature data [14], but containing a more complex mixture than pure HT, named A009. We investigated whether A009 was able to interfere with angiogenesis in vitro and in vivo. The anti-angiogenic and angiopreventive potential of A009 was compared with that of HT at similar concentrations.

The A009 extract exerted greater inhibition of endothelial cell proliferation, migration and invasion. Inhibition of cell proliferation was associated with the induction of apoptosis in treated HUVECs. HT showed a similar effect as A009 on endothelial cell morphogenesis. Given the previously reported anti-oxidant activity of HT, we examined the ability of A009 to inhibit ROS generation, compared to HT alone. We found that A009 exerted a more potent ROS scavenger activity than that of HT alone, either in a preventive or in a protective schedule. A009 was able to decrease the generation of ROS as a pre-treatment 
Citation: Rossi T, Bassani B, Gallo C, Maramotti S, Noonan DM, et al. (2015) Effect of a Purified Extract of Olive Mill Waste water on Endothelial Cell Proliferation, Apoptosis, Migration and Capillary-Like Structure in vitro and in vivo. J Bioanal Biomed S12: 006. doi:10.4172/1948-593X. S12-006

Page 7 of 8

or when administered after reactive species induction through $\mathrm{H}_{2} \mathrm{O}_{2}$. These data clearly support the hypothesis of a significant scavenger activity exerted by A009. Both A009 and HT were able to interfere with the ability of HUVECs to form capillary-like structures in vitro.

Finally, using the matrigel sponge assay, we demonstrated that A009 inhibited angiogenesis in vivo, as indicated by the decreased hemoglobin content in plugs excised from treated mice. The antiangiogenic activity of A009 was more potent in vivo compared to HT alone. Altogether, our data demonstrate that A009, a highly phenolicrich extract from OMWW, shows a significant anti-angiogenic and angiopreventive potential.

The use of two different batches, characterized by a different phenolic composition, demonstrated that the A009 complex, independently from the minimal variation in composition, exerts a stronger anti-angiogenic effect compared to hydroxytyrosol alone. This feature suggests that the A009 mixture has additional components that are additive/synergistic as compared to commercially available hydroxytyrosol. The differences in batch composition indicate that further investigation of the phenolic components distinguishing the different batches could shed light on the specific activity of specific components and indicate which cultivars might produce a greater effect, as olive oil phenols have been found to vary between cultivars [35]. Interestingly, the 2 batches varied widely in the presence of secoiridoid derivatives (29\% in batch 1 and $4.7 \%$ in batch 2 ; Table S1). Secoiridoid derivatives, in particular (-)-oleocanthal, has shown effects as a $c$-met inhibitor [36,37]. The met pathway represents an additional VEGF independent mechanism of tumor angiogenesis, and inhibition of met inhibits tumor growth that correlates with repression of angiogenesis [38]. Taken together, our data demonstrated the antiangiogenic and angio-preventive activity in vitro and in vivo of a novel phenols enriched extract showing promising application for preventive approaches/strategies, recovering a waste material.

\section{Acknowledgement}

TR and BB are students of the PhD program in Biotechnology, Biosciences and Surgical Technologies, School in Biological and Medical Sciences, University of Insubria. AB was a FIRC (Fondazione Italiana per la Ricerca sul Cancro) fellow. CG is student of the PhD program in Biotechnologies and Biosciences, University of Parma. This study was initiated and supported by a grant from the Fattoria La Vialla di Gianni, Antonio e Bandino Lo Franco -SAS. We are grateful to Massimo and Daniele Pizzichini (General Lab srl, ENEA) for the A009 extracts. We are grateful to Paola Corradino for assistance and bibliography and Alessandra Panvini Rosati for secretarial help.

\section{References}

1. Prinelli F, Yannakoulia M, Anastasiou CA, Adorni F, Di Santo SG, et al. (2015) Mediterranean diet and other lifestyle factors in relation to 20-year all-cause mortality: a cohort study in an Italian population. Br J Nutr 113: 1003-1011.

2. InterAct Consortium (2014) Adherence to predefined dietary patterns and incident type 2 diabetes in European populations: EPIC-InterAct Study. Diabetologia 57: 321-333

3. Widmer RJ, Flammer AJ, Lerman LO, Lerman A (2015) The Mediterranean diet, its components, and cardiovascular disease. Am J Med 128: 229-238.

4. Schwingshackl L, Hoffmann G (2014) Adherence to Mediterranean diet and risk of cancer: a systematic review and meta-analysis of observational studies. Int J Cancer 135: 1884-1897.

5. Kontou N, Psaltopoulou T, Panagiotakos D, Dimopoulos MA, Linos A (2011) The mediterranean diet in cancer prevention: a review. J Med Food 14 1065-1078.

6. Schwingshackl L, Hoffmann G (2014) Adherence to Mediterranean diet and risk of cancer: a systematic review and meta-analysis of observational studies. Int J Cancer 135: 1884-1897.
7. Albuquerque RC, Baltar VT, Marchioni DM (2014) Breast cancer and dietary patterns: a systematic review. Nutr Rev 72: 1-17.

8. Giacosa A, Barale R, Bavaresco L, Gatenby P, Gerbi V, et al. (2013) Cancer prevention in Europe: the Mediterranean diet as a protective choice. Eur $\mathrm{J}$ Cancer Prev 22: 90-95.

9. Casaburi I, Puoci F, Chimento A, Sirianni R, Ruggiero C, et al. (2013) Potentia of olive oil phenols as chemopreventive and therapeutic agents against cancer: a review of in vitro studies. Mol Nutr Food Res 57: 71-83.

10. Sporn MB, Liby KT (2005) Cancer chemoprevention: scientific promise, clinical uncertainty. Nat Clin Pract Oncol 2: 518-525.

11. Sporn MB, Suh N (2002) Chemoprevention: an essential approach to controlling cancer. Nat Rev Cancer 2: 537-543.

12. Li WW, Li VW, Hutnik M, Chiou AS (2012) Tumor angiogenesis as a target for dietary cancer prevention. J Oncol 2012: 879623.

13. Fassina G, Venè R, Morini M, Minghelli S, Benelli R, et al. (2004) Mechanisms of inhibition of tumor angiogenesis and vascular tumor growth by epigallocatechin3-gallate. Clin Cancer Res 10: 4865-4873.

14. Schaffer S, Müller WE, Eckert GP (2010) Cytoprotective effects of olive mill wastewater extract and its main constituent hydroxytyrosol in PC12 cells. Pharmacol Res 62: 322-327.

15. Achmon Y, Fishman A (2015) The antioxidant hydroxytyrosol: biotechnologica production challenges and opportunities. Appl Microbiol Biotechnol 99: 1119-1130.

16. Burattini S, Salucci S, Baldassarri V, Accorsi A, Piatti E, et al. (2013) Antiapoptotic activity of hydroxytyrosol and hydroxytyrosyl laurate. Food Chem Toxicol 55: 248-256

17. Bernini R, Merendino N, Romani A, Velotti F (2013) Naturally occurring hydroxytyrosol: synthesis and anticancer potential. Curr Med Chem 20: 655-670.

18. Ranalli A, Lucera L, Contento S (2003) Antioxidizing potency of pheno compounds in olive oil mill wastewater. J Agric Food Chem 51: 7636-7641.

19. Poerschmann J, Weiner B, Baskyr I (2013) Organic compounds in olive mill wastewater and in solutions resulting from hydrothermal carbonization of the wastewater. Chemosphere 92: 1472-1482.

20. Frankel E, Bakhouche A, Lozano-Sanchez J, Segura-Carretero A, FernandezGutierrez A (2013) Literature review on production process to obtain extra virgin olive oil enriched in bioactive compounds. Potential use of byproducts as alternative sources of polyphenols. J Agric Food Chem 61: 5179-88.

21. Niaounakis M, Halvadakis CP (2006) Olive-mill Waste Management. (2 editon) ypothito, Athens, Greece

22. Cárdeno A, Sánchez-Hidalgo M, Alarcón-de-la-Lastra C (2013) An up-date of olive oil phenols in inflammation and cancer: molecular mechanisms and clinical implications. Curr Med Chem 20: 4758-4776.

23. Tosetti F, Ferrari N, De Flora S, Albini A (2002) Angioprevention': angiogenesis is a common and key target for cancer chemopreventive agents. FASEB $J$ 16: $2-14$.

24. Hanahan D, Weinberg RA (2011) Hallmarks of cancer: the next generation Cell 144: 646-674.

25. Licznerska BE, Szaefer H, Murias M, Bartoszek A, Baer-Dubowska W (2013) Modulation of CYP19 expression by cabbage juices and their active components: indole-3-carbinol and ,3'-diindolylmethene in human breast epithelial cell lines. Eur J Nutr 52: 1483-1492.

26. Albini A, Tosetti F, Li VW, Noonan DM, Li WW (2012) Cancer prevention by targeting angiogenesis. Nat Rev Clin Oncol 9: 498-509.

27. Vené R, Delfino L, Castellani P, Balza E, Bertolotti M, et al. (2010) Redox remodeling allows and controls $\mathrm{B}$-cell activation and differentiation. Antioxid Redox Signal 13: 1145-1155

28. Albini A, Iwamoto Y, Kleinman HK, Martin GR, Aaronson SA, et al. (1987) A rapid in vitro assay for quantitating the invasive potential of tumor cells. Cancer Res 47: 3239-3245.

29. Albini A, Benelli R (2007) The chemoinvasion assay: a method to assess tumo and endothelial cell invasion and its modulation. Nat Protoc 2: 504-511.

30. Grant DS, Tashiro K, Segui-Real B, Yamada Y, Martin GR, et al. (1989) Two 
Citation: Rossi T, Bassani B, Gallo C, Maramotti S, Noonan DM, et al. (2015) Effect of a Purified Extract of Olive Mill Waste water on Endothelial Cell Proliferation, Apoptosis, Migration and Capillary-Like Structure in vitro and in vivo. J Bioanal Biomed S12: 006. doi:10.4172/1948-593X. S12-006

Page 8 of 8

different laminin domains mediate the differentiation of human endothelial cells into capillary-like structures in vitro. Cell 58: 933-943.

31. Albini A, Fontanini G, Masiello L, Tacchetti C, Bigini D, et al. (1994) Angiogenic potential in vivo by Kaposi's sarcoma cell-free supernatants and HIV-1 tat product: inhibition of KS-like lesions by tissue inhibitor of metalloproteinase-2. AIDS 8: 1237-1244.

32. Rossi T, Gallo C, Bassani B, Canali S, Albini A, et al. (2014) Drink your prevention: beverages with cancer preventive phytochemicals. Pol Arch Med Wewn 124: 713-722

33. Tosetti F, Noonan DM, Albini A (2009) Metabolic regulation and redox activity as mechanisms for angioprevention by dietary phytochemicals. Int $\mathrm{J}$ Cancer 125: $1997-2003$

34. Covas MI (2008) Bioactive effects of olive oil phenolic compounds in humans: reduction of heart disease factors and oxidative damage. Inflammopharmacology 16: 216-218.

35. Sánchez de Medina V, Priego-Capote F, de Castro MD1 (2015) Characterization of monovarietal virgin olive oils by phenols profiling. Talanta 132: 424-432.

36. Akl MR, Ayoub NM, Mohyeldin MM, Busnena BA, Foudah Al, et al. (2014) Olive phenolics as c-Met inhibitors: (-)-Oleocanthal attenuates cell proliferation, invasiveness, and tumor growth in breast cancer models. PLoS One 9: e97622.

37. Elnagar AY, Sylvester PW, El Sayed KA (2011) (-)-Oleocanthal as a c-Met inhibitor for the control of metastatic breast and prostate cancers. Planta Med 77: 1013-1019.

38. Cantelmo AR, Cammarota R, Noonan DM, Focaccetti C, Comoglio PM, et al. (2010) Cell delivery of Met docking site peptides inhibit angiogenesis and vascular tumor growth. Oncogene 29: 5286-5298.
This article was originally published in a special issue, Development of Phytochemicals as Drugs handled by Editor. Dr. Erik de Leeuw, University of Maryland, USA 